\title{
Assessment of Non-Contact Measurement Using a Microwave Sensor to Jugular Venous Pulse Monitoring
}

\author{
Satoshi Suzuki ${ }^{1}$, Masaaki Hoshiga ${ }^{2}$, Kentaro Kotani $^{1}$, Takafumi Asao ${ }^{1}$ \\ ${ }^{1}$ Faculty of Engineering Science, Kansai University, Osaka, Japan; ${ }^{2}$ Faculty of Medicine, Osaka Medical College, \\ Osaka, Japan \\ Correspondence to: Satoshi Suzuki, ssuzuki@kansai-u.ac.jp \\ Keywords: Microwave Radar, Non-Contact Monitoring, Jugular Venous Pressure, Right Heart Function \\ Received: January 25, $2021 \quad$ Accepted: March 7, $2021 \quad$ Published: March 10, 2021
}

Copyright $\odot 2021$ by author(s) and Scientific Research Publishing Inc.

This work is licensed under the Creative Commons Attribution International License (CC BY 4.0).

http://creativecommons.org/licenses/by/4.0/

\section{(c) (i) Open Access}

\section{ABSTRACT}

The jugular venous pulse (JVP) waveform provides an insight into right heart function, and its assessment is important in patients with heart failure. However, the conventional pulse-transducer (contact) method for monitoring this waveform is not frequently used because it requires a high degree of skill. The aim of this study was to confirm the effectiveness of a prototype non-contact system that employs microwave radar $(24 \mathrm{GHz}, 7 \mathrm{~mW}$; non-contact system) for JVP measurement. Experiments were conducted on eight healthy male volunteers $(21.88 \pm 0.99$ years). JVP measurements were compared between the conventional contact method and the proposed non-contact method. Change in JVP waveform was measured in response to an angle of reclining in five steps from the supine position to $75^{\circ}$ of elevation. The obtained JVP measurements were similar between the two methods. Because in the non-contact method the faint pulsation of the JVP is not suppressed by the pressure of a sensor placed on the skin, the prototype microwave radar system is particularly suitable for evaluating the JVP waveform.

\section{INTRODUCTION}

Numerous studies have investigated the left ventricle from morphological and functional viewpoints; in contrast, there are few studies regarding the right ventricle. It is considered that the symptoms of left heart failure can worsen rapidly with pulmonary congestion, but many of the symptoms of right heart failure, due to congestion of the venous system, are slow in onset [1], and take time to investigate and diagnose. In addition, the structure and physiological character of the right ventricle are more complicated than those of the left ventricle. More attention has recently been paid to the right ventricle with respect to its role in heart failure; e.g., in evaluation of the relationship between right heart failure and indices of exercise tolerability and prognosis [1-3]. 
Ultrasonographic techniques proposed for the non-invasive evaluation of right ventricular systolic function include the 2D speckle tracking method [4,5] and 3D ultrasonographic method [6]. However, no reliable non-invasive method for evaluation of right ventricular diastolic function has yet emerged. Evaluation of right ventricular diastolic function is generally by measurement of right atrial pressure (RAP) [1, 2], which requires placement of a central venous catheter or Swan-Ganz catheter, which is invasive and places a heavy burden on the patient $[7,8]$.

A previous study has noted that it is possible to evaluate right ventricular diastolic function non-invasively by monitoring jugular venous pulse (JVP) $[9,10]$. The JVP can accurately reflect change in right atrial pressure because in contrast to other veins, the jugular veins have only one pair of valves, which are situated upward from the right atrium. Although the JVP value may have significance similar to that of RAP, this has not been proven because JVP is not in common clinical use, for the reason that its measurement requires considerable skill [11]. It is thought that jugular venous pressure is only $4-6$ $\mathrm{mmHg}[12,13]$. When the point for measurement of JVP is identified by palpation, the jugular vein is compressed by the fingers, and it becomes difficult to find pulsation of the vein. JVP information is revealed in the peaks and valleys of each heartbeat but the values are very small and easily lost; accordingly, JVP is considered an inefficient and unsuitable index. This is also a problem in contact sensing. JVP can be monitored using a diaphragm sensor that must be set on a specific area just superficial to the jugular vein in the right neck, because the scale of motion induced on the body surface by JVP is so small. Therefore, sensors for measuring JVP must be highly sensitive to enable acquisition of signal of such small magnitude without compressing the jugular vein.

In a new development, a method for monitoring vital signs using microwaves has recently been proposed [14-16]. The method monitors vital signs non-invasively without directly touching the body, and can measure motion induced on the body surface by cardiac and respiratory activity on an extremely small scale. The measurement area can be controlled by the antenna settings. A previous study has reported practical uses for this technique such as a ceiling-mounted microwave radar, positioned approximately 2 $\mathrm{m}$ above the subject, that can monitor the patient's respiratory rate through thick bedding. Monitoring of HRV (heart rate variability) has also been achieved $[17,18]$. The results of these studies indicate that the circulatory system can be monitored using this technique. As the characteristics of the microwave radar method correspond to the requirements for measurement of JVP, the method could possibly be applied for this use, and would avoid placing a heavy physiological burden on the patients.

Therefore, the aim of the present research was to confirm the effectiveness of a non-contact technique that uses microwave radar for measurement of JVP. We developed a prototype system for this purpose and verified its effectiveness utilizing experimental data. We also discuss the potential of the method for future application.

\section{SYSTEM DESIGN}

\subsection{Microwave Radar Sensor and Specifications}

The prototype system is generally equivalent to a system described previously $[15,18]$, comprising a microwave radar sensor, patch antenna, and analyzer (Figure 1). The small microwave radar sensor (NJR4261J; New Japan Radio Co., Ltd., Tokyo, Japan) has continuous-wave carrier frequency of $24 \mathrm{GHz}$ and mean output power of $7 \mathrm{~mW}$ (maximum $10 \mathrm{~mW}$ ). The patch antenna has gain of $10 \mathrm{dBi}$ and diffusion angle of approximately 40 degrees. According to the guidelines for radio waves established by the Telecommunication Bureau of the Ministry of Internal Affairs and Communication of Japan [19], electrical field intensity is limited to $1 \mathrm{~mW} / \mathrm{cm}^{2}$ at frequencies $>3 \mathrm{GHz}$. The electrical field intensity of the microwave radar is $0.7 \mathrm{~mW} / \mathrm{cm}^{2}$, thus conforming to the guidelines. The frequency band of our device is within the limits of normal use for radio waves approved by Japanese law.

\subsection{Measurement Data and Indices for Estimation of JVP}

The output signals from two microwave radar sensors were amplified 50-fold by a DC amplifier 


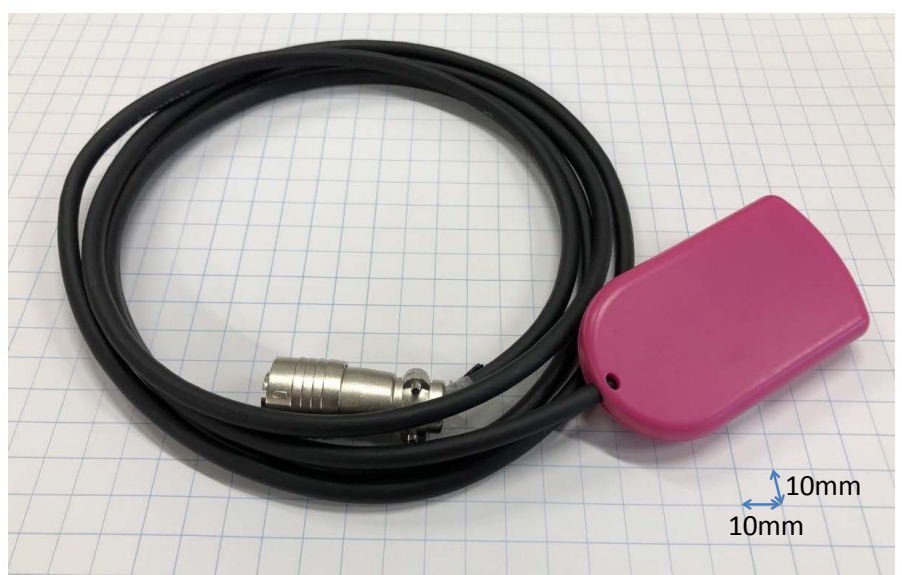

Figure 1. Prototype non-contact monitoring system for observing JVP, comprising a microwave radar sensor and patch antenna. The size of the microwave radar sensor is $45 \times 22 \times 10 \mathrm{~mm}$ (the housing size is $85 \times 48 \times 15 \mathrm{~mm}$ ); transmit/receive, the carrier frequency is $24 \mathrm{GHz}$ with continuous wave, and mean output power was $7 \mathrm{~mW}$ (maximum, $10 \mathrm{~mW}$ ) with $10 \mathrm{dBi}$ patch antenna.

(DA-710A; Kyowa Electronic Instruments Co., Ltd., Tokyo, Japan). The output signals from reference outputs and two microwave radars were sampled by an A/D converter (USB-6211; National Instruments Co., Texas, US) with a sampling interval of $1 \mathrm{~ms}$ and stored in real time on a PC with system controls written in LabVIEW (National Instruments). After A/D converter sampling, 0.5 and $10 \mathrm{~Hz}$ bandpass filters were used to reduce noise and interference in both radar outputs. JVP normally has 3 or 4 fluctuations per cyclic oscillation, corresponding to one heartbeat. The heart rate in the normal state is $1-2 \mathrm{~Hz}$. Therefore, the bandpass filters adequately covered a range of 3 - 4 fluctuations per heartbeat. After bandpass filtering, the JVP parameters were acquired from the signal data and compared with those acquired by the reference device.

\section{METHODS}

\subsection{Experimental Setting and Protocols}

The experiments were conducted on eight healthy male volunteer subjects (age range, 21 - 24 years; $(21.88 \pm 0.99$ years $)$ for approximately one hour in the midafternoon. Subjects without a history of serious illness were selected for this study and instructions regarding the experiment were given and informed consent was obtained. The study was conducted with the cooperation of Kansai Medical College and the study protocol was reviewed and approved by the Committee on Human Research of Kansai Medical College.

The reference data were obtained first, with placement of ECG electrodes and a diaphragm pressure sensor on the subject's body. Precordial ECG (Biotop; NEC Sanei Co., Tokyo, Japan) was obtained with the electrodes placed in the V5 position. JVP was measured using a diaphragm pressure sensor (MES-1000; Fukuda Denshi Co., Ltd., Tokyo, Japan) placed directly on the subject's internal jugular vein. The position of the internal jugular vein was confirmed by a medical specialist prior to each experiment, and the position was identified by ultrasound (Xario 200; Canon Medical Systems Inc., Tokyo, Japan).

In the prototype experiment, the microwave radar sensor was placed directly above the subject's internal jugular vein. A $12.5 \mathrm{~mm}$ guide spacer was installed on the sensor housing to maintain a distance of one wavelength of $24 \mathrm{GHz}$ microwaves between the housing and the skin surface.

In the clinical setting, JVP measurements are usually obtained by the diaphragm pressure sensor method with the patient reclining at an angle of $45^{\circ}[12,13]$. In the experiments, for both methods we measured change in JVP waveform in response to change in angle of recline of the patient bed, as described be- 
low.

The control data were obtained as follows: after placement of electrodes, the subject was positioned supine on an adjustable bed (KA-55151M; Paramount Bed Co., Tokyo, Japan) and allowed to rest for several minutes. JVP was then measured over a period of one minute. JVP was measured with each of the contact and the non-contact sensing methods in six conditions in various degrees of recline, from fully supine $\left(0^{\circ}\right.$, control $)$ to $75^{\circ}$ in increments of $15^{\circ}$, each over a period of one minute. To compare the two sensing methods, ideally the two sensors would both have been placed directly over the internal jugular vein at the same time, and measured synchronously. However, this was physically impossible because the sensors overlapped, and therefore placement and measurement were performed alternately with the diaphragm pressure sensor and microwave radar sensor (Figure 2(b)).

\subsection{Analytical Protocols of Acquired Data}

The JVP waveform comprises an a-wave, c- wave, v-wave, $\mathrm{x}$-valley and y-valley in each beat. To confirm the accuracy of the signal acquired by the non-contact method, we compared the occurrence times and amplitudes of a- and v-wave, and also $\mathrm{x}$ - and $\mathrm{y}$-valley in each beat between the two methods. We did not analyze the c-wave because it is often not distinguishable in the JVP [20].

The MES-1000 system (reference diaphragm pressure sensor system) has no ports to output analog signals and cannot store digital signal data. Therefore, we captured and stored each frame that displayed a measurement and extracted the shapes of the output signals by image processing. We also used image processing to extract the coordinates of the peak points in the $\mathrm{x}$ and $\mathrm{y}$ axes of each wave and valley for a-wave, $v$-wave, $x$-valley, and $y$-valley from the images displayed on the screen. Similarly, for the data acquired from the microwave radar sensor, only the AC component was extracted for analysis. The amplitudes of the cyclic oscillations corresponding to each heartbeat and the amplitude values and generated times of the a- and $\mathrm{v}$-waves and $\mathrm{x}$ - and $\mathrm{y}$-valleys generated for each heartbeat were extracted for comparison with the reference values.

To compare the two methods, we compared the normalized coordinate data for each wave and valley in the time and amplitude directions. It was difficult to directly compare the data obtained by the two measurement methods because the timing of the data was not synchronized between them and there were slight differences between the heartbeats in each measurement period. Statistical analysis was performed using Excel and non-contact method was evaluated by the correlation coefficient value and the p-value calculated by the paired t-test.

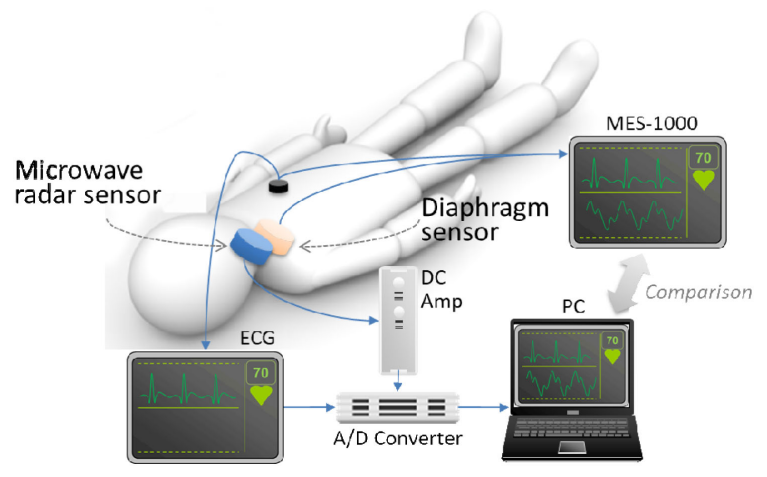

(a)

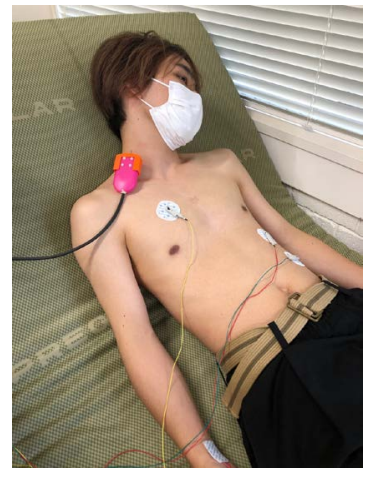

(b)

Figure 2. Measurement of JVP waveform at various angles of recline. (a) Schema of the experimental setup. (b) Photograph showing the upper body in recline on the bed. JVW was measured by each method in each position from fully supine (control) to $75^{\circ}$ of incline in steps of $15^{\circ}$ over a period of one minute. 


\section{RESULTS}

Figure 3(a) shows a representative JVP waveform obtained by the diaphragm pressure sensor system for a period of about five seconds. Cyclic oscillations in the JVP waveform are similar to those of an ECG waveform and show long-period fluctuations in baseline due to respiratory fluctuations. All waveform components can be clearly identified. Figure 3(b) shows a representative JVP trace obtained by the microwave radar sensor system in the same subject for a period of about five seconds. Although the amplitudes of the $x$ - and $y$-valleys differ from those shown in Figure 3(a), cyclic oscillation is apparent and the waveform is similar to that for the diaphragm pressure sensor system. Similar waveform characteristics could be observed in all subjects. These results confirm similarity between JVP data and measurements obtained by the prototype non-contact method microwave radar system and those obtained by the contact method using the diaphragm pressure sensor.

Figure 4 shows the comparison of normalized coordinates in the amplitude direction of the JVP a-wave, $v$-wave, $x$-valley and $y$-valley values of all subjects in the condition of reclining angle of $0^{\circ}$ (supine position). These values show the positional relationships of all waves and valleys within one full JVP waveform. Data obtained by the non-contact method were consistent with the reference data measured by the contact method $(r=0.895, p=0.0019)$; i.e., the non-contact measurement system using microwave radar could accurately grasp characteristic JVP information.

Figure 5 shows mean JVP amplitude between the $\mathrm{x}$-valley and a-wave for the two measurement systems for reclining angles of $0^{\circ}-75^{\circ}$ in all subjects. Amplitude values differed slightly between the two methods but showed a similar decreasing tendency with increasing reclining angle. As the reclining angle increased, the internal jugular vein became more vertical and the propagation of pressure from the right atrium through the internal jugular vein decreased. Accordingly, pulsation of the internal jugular vein decreased and the amplitudes measured by both methods also decreased. The similar results obtained in both measurement systems indicate the validity of information obtained by the non-contact measurement system.

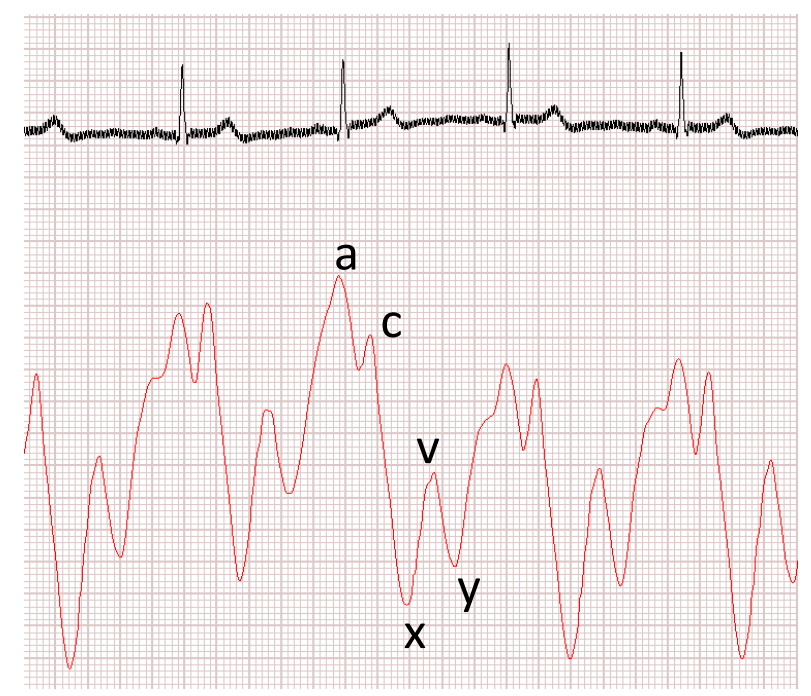

(a)

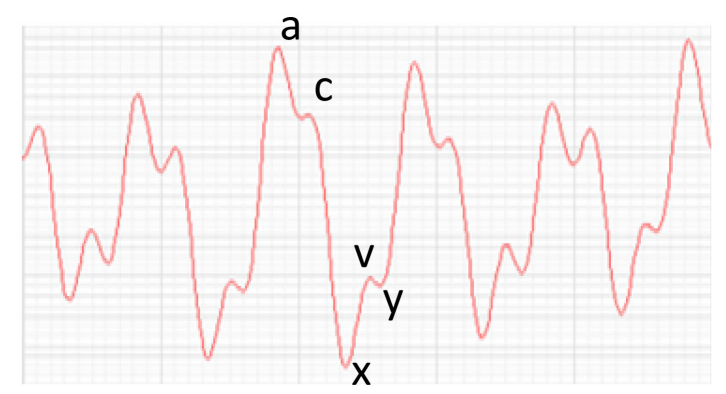

(b)

Figure 3. Sample signals acquired by the reference devices (ECG and diaphragm) and the prototype system. (a) Sample ECG (upper part), and JVP signal (lower part) measured by the diaphragm pressure sensor system in subject A over a period of 5 seconds. (b) Sample JVP signal measured by the non-contact system in subject $A$ over a period of 5 seconds. The lower-case letters indicate the a-wave, $\mathrm{c}$-wave, $\mathrm{v}$-wave, $\mathrm{x}$-valley, and $\mathrm{y}$-valley components of the JVP waveform. 


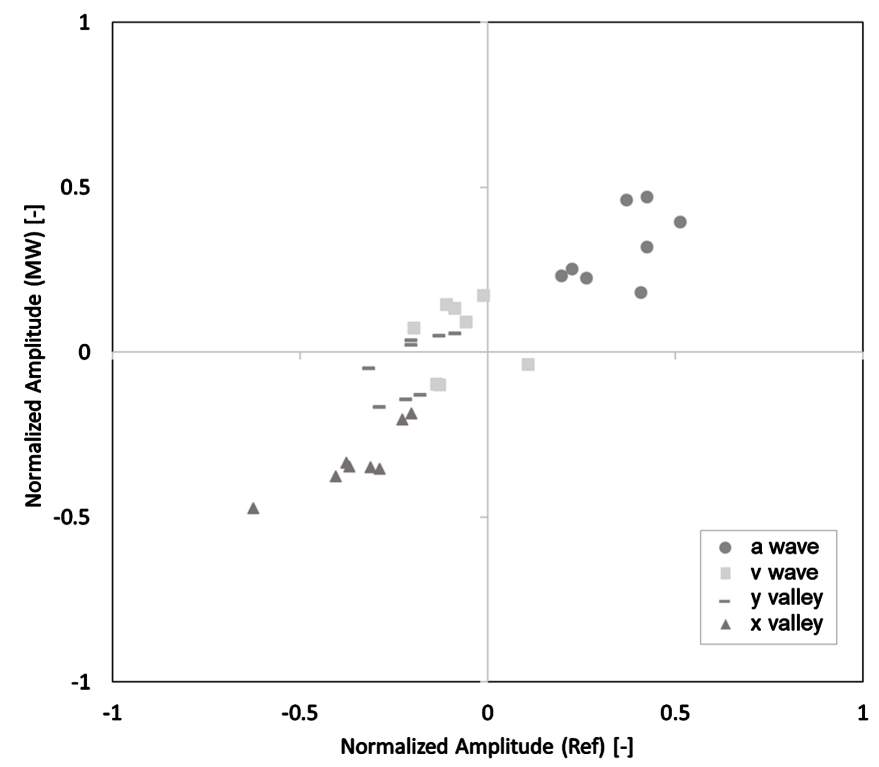

Figure 4. Comparison of JVP measured by the diaphragm sensor and microwave radar sensor methods at a reclining angle of $0^{\circ}$. Normalized amplitudes of all beats for all subjects obtained within the same measurement time for the diaphragm sensor (horizontal axis) and microwave radar sensor (vertical axis) show a clear and stable linear relation $(r=0.895, p=0.0019)$.

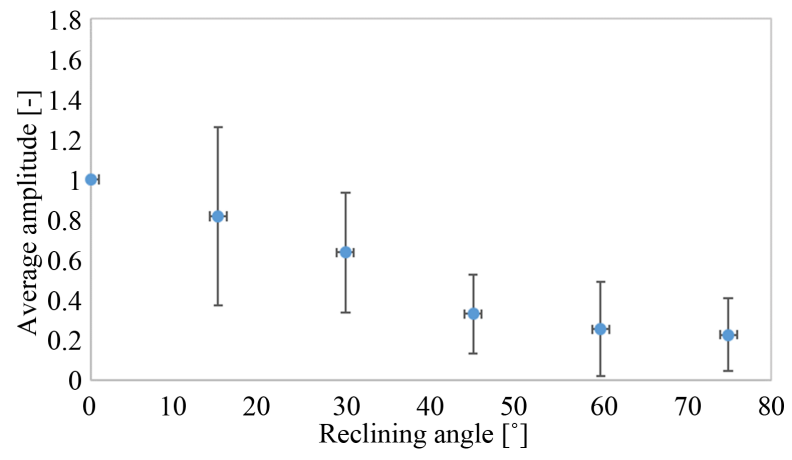

(a)

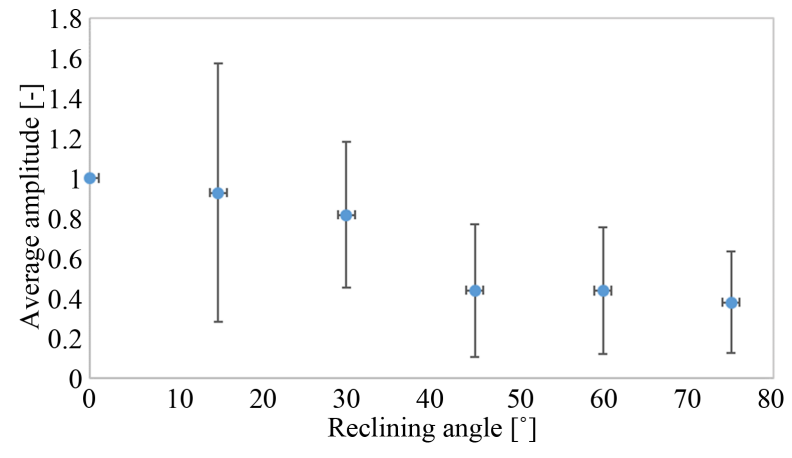

(b)

Figure 5. JVP amplitude according to reclining angle. Mean amplitude between the $\mathrm{x}$-valley and a-wave components of the JVP waveform for all patients is shown for measurement by the (a) diaphragm sensor and (b) microwave radar sensor systems. In both measurement methods, the amplitude of JVP output shows a tendency to decrease as the reclining angle increases. Therefore, it is highly likely that JVP is captured by microwave radar.

\section{DISCUSSION}

The results of the experiments confirmed the effectiveness of the non-contact method using microwave radar for measurement of JVP. To obtain measurements under strict conditions, we set the microwave radar sensor at a distance from the skin equal to that of a standing wave of $24 \mathrm{GHz}$ microwaves; i.e., $12.5 \mathrm{~mm}$. However, we confirmed that pulsation on the skin surface could be measured from more distal points, from several centimeters to $\sim 10$ centimeters i.e., about 10 wavelength of $24 \mathrm{GHz}$ microwave [14, 15]. Therefore, this system has potential to obtain measurements from distances more remote from the skin. 
In conventional measurement of right ventricular systolic function by JVP, the RAP value is very small ( $\sim 5 \mathrm{mmHg})$. Therefore, it is highly likely that the pressure of contact sensors set directly on the body surface and of palpation by fingers near the area of the internal jugular vein will change the shape of the JVP waveform and prevent detection of this information. In other words, the contact method is basically unsuitable for measurement of JVP. In contrast, motion on the surface of the body is not inhibited when JVP is measured by the microwave radar sensor system. In the present study, the proposed microwave radar sensor method acquired a-wave, $v$-wave, $x$-valley and y-valley JVP information in all subjects. The high success rate of the proposed system is likely due to the inherent suitability of a non-contact acquisition and monitoring method in the situation of high sensitivity to pressure on the skin surface.

A recent study has reported that the JVP waveform contains important prognostic information regarding heart failure $[1,21]$. We expect that use of the proposed method in acquisition of accurate JVP waveform information will contribute to the diagnosis of such pathologies.

The proposed non-contact method has some advantages compared with the conventional method. Although not completely uniform, the detection area of the proposed system is wider than with the diaphragm sensor system. This advantage increases the usability of the system for medical staff in terms of eliminating the time required to position the sensor. The system also has the advantage of reduced patient burden as it is completely non-contact and non-invasive.

Disadvantages of the proposed system include that the deviation tended to be slightly larger as the reclining angle increased. We consider that pressure is less likely to be propagated through the internal jugular vein as the reclining angle increases and the upper body becomes more upright because the right atrial pressure is originally low and decreases further with increasing reclining angle. Body fat mass also affects propagation of the JVP pulse to the body surface. Therefore, it is necessary to conduct further investigations that take personal physical characteristics into consideration. We did not consider the effects of artifacts due to such as the contribution of arterial information, which could possibly be eliminated with use of an algorithm. Because we could not perform synchronous measurement with the two sensors set at the same position, there was a small time lag between the two datasets, which reduced the accuracy of the comparisons. In future study, the accuracy of the proposed method could be further assessed by conducting a comparison with catheter-based measurements.

\section{CONCLUSIONS}

Despite several limitations, assessment of JVP measurements obtained by a non-contact method using microwave radar sensors showed the potential of the technique and its higher accuracy compared with the conventional contact method.

Further evaluation of the method in real patients is necessary to assess its suitability for medical application and possible usefulness for prognosis in heart failure.

\section{ACKNOWLEDGEMENTS}

This research received financial support from Kansai University Fund for Domestic and Overseas Research, 2018.

\section{CONFLICTS OF INTEREST}

The authors declare no conflicts of interest regarding the publication of this paper.

\section{REFERENCES}

1. Harada, D., Aasanoi, H., Ushijima, R., Noto, T., Takagawa, J., Ishise, H. and Inoue, H. (2018) Impact of Right Ventricular Distensibility on Congestive Heart Failure with Preserved Left Ventricular Ejection Fraction in the Elderly. Heart and Vessels, 33, 605-614. https://doi.org/10.1007/s00380-017-1092-2

2. Taniguchi, T., Sakata, Y., Ohtani, T., Mizote, I., Takeda, Y., Asano, Y., Masuda, M., Minamiguchi, H., Kanzaki, 
M., Ichibori, Y., Nishi, H., Toda, K., Sawa, Y. and Komuro, I. (2014) Usefulness of Transient Elastography for Noninvasive and Reliable Estimation of Right-Sided Filling Pressure in Heart Failure. American Journal of Cardiology, 113, 552-558. https://doi.org/10.1016/j.amjcard.2013.10.018

3. De Groote, P., Millaire, A., Foucher-Hossein, C., Nugue, O., Marchandise, X., Ducloux, G. and Lablanche, J.M. (1998) Right Ventricular Ejection Fraction Is an Independent Predictor of Survival in Patients with Moderate Heart Failure. Journal of the American College of Cardiology, 32, 948-954. https://doi.org/10.1016/S0735-1097(98)00337-4

4. Bohs, L.N., Geiman, B.J., Anderson, M.E., Gebhart, S.C. and Trahey, G.E. (2000) Speckle Tracking for Multi-Dimensional Flow Estimation. Ultrasonics, 38, 369-375. https://doi.org/10.1016/S0041-624X(99)00182-1

5. Pavlopoulos, H. and Nihoyannopoulos, P. (2008) Strain and Strain Rate Deformation Parameters: From Tissue Doppler to 2D Speckle Tracking. International Journal of Cardiovascular Imaging, 24, 479-491. https://doi.org/10.1007/s10554-007-9286-9

6. Yagel, S., Cohen, S.M., Shapiro, I. and Valsky, D.V. (2007) 3D and 4D Ultrasound in Fetal Cardiac Scanning: A New Look at the Fetal Heart. Ultrasound in Obstetrics and Gynecology, 29, 81-95.

https://doi.org/10.1002/uog.3912

7. Jagger, J., Perry, J., Parker, G. and Phillips, E.K. (2011) Nursing 2011 Survey Results: Blood Exposure Risk during Peripheral I.V. Catheter Insertion and Removal. Nursing, 41, 45-49.

https://doi.org/10.1097/01.NURSE.0000407678.81635.62

8. Green, B. and Griffiths, E.C. (2013) Psychiatric Consequences of Needlestick Injury. Occupational Medicine, 63, 183-188. https://doi.org/10.1093/occmed/kqt006

9. McGee, S.R. (1998) Physical Examination of Venous Pressure: A Critical Review. American Heart Journal, 136, 10-18. https://doi.org/10.1016/S0002-8703(98)70175-9

10. Braganza, M., Shaw, J., Solverson, K., Vis, D., Janovcik, J., Varughese, R.A., Thakrar, M.V., Hirani, N., Helmersen, D. and Weatherald, J. (2019) A Prospective Evaluation of the Diagnostic Accuracy of the Physical Examination for Pulmonary Hypertension. Chest, 155, 982-990. https://doi.org/10.1016/j.chest.2019.01.035

11. Metkus, T.S. and Kim, B.S. (2015) Bedside Diagnosis in the Intensive Care Unit: Is Looking Overlooked? Annals of the American Thoracic Society, 12, 1447-1450. https://doi.org/10.1513/AnnalsATS.201505-271OI

12. Applefeld, M.M. (1990) The Jugular Venous Pressure and Pulse Contour. In: Walker, H.K., Hall, W.D. and Hurst, J.W., Eds., Clinical Methods. The History, Physical, and Laboratory Examinations, 3rd Edition, Butterworths, Boston, Chapter 19.

13. Gilreath, A., DeVesty, G., Stone, D., Lee Allen, C. and Pravikoff, D. (2016) Nursing Practice and Skill-Jugular Venous Pressure: Measuring. CINAHL Information Systems, Glendale, 1-4.

https://www.ebscohost.com/assets-sample-content/NRC NPS Measuring_Jugular_Venous Pressure.pdf

14. Suzuki, S., Matsui, T., Kawahara, H., Ichiki, H., Shimizu, J., Kondo, Y., Gotoh, S., Yura, H., Takase, B. and Ishihara, M. (2009) A Non-Contact Vital Sign Monitoring System for Ambulances Using Dual-Frequency Microwave Radars. Medical and Biological Engineering and Computing, 47, 101-105.

https://doi.org/10.1007/s11517-008-0408-x

15. Suzuki, S., Matsui, T., Kagawa, M., Asao, T. and Kotani, K. (2013) An Approach to a Non-Contact Vital Sign Monitoring Using Dual-Frequency Microwave Radars for Elderly Care. Journal of Biomedical Science and Engineering, 6, 704-711. https://doi.org/10.4236/jbise.2013.67086

16. Sun, G., Matsui, T., Watai, Y., Kim, S., Kirimoto, T., Suzuki, S. and Hakozaki, Y. (2018) Vital-SCOPE: Design and Evaluation of a Smart Vital Sign Monitor for Simultaneous Measurement of Pulse Rate, Respiratory Rate and Body Temperature for Patient Monitoring. Journal of Sensors, 2018, Article ID: 4371872. https://doi.org/10.1155/2018/4371872 
17. Suzuki, S., Matsui, T., Sugawara, K., Asao, T. and Kotani, K. (2011) An Approach to Remote Monitoring of Heart Rate Variability (HRV) Using Microwave Radar during a Calculation Task. Journal of Physiological Anthropology, 30, 241-249. https://doi.org/10.2114/jpa2.30.241

18. Suzuki, S., Matsui, T., Imuta, H., Uenoyama, M., Yura, H., Ishihara, M. and Kawakami, M. (2008) A Novel Autonomic Activation Measurement Method for Stress Monitoring: Non-Contact Measurement of Heart Rate Variability Using a Compact Microwave Radar. Medical and Biological Engineering and Computing, 46, 709-714. https://doi.org/10.1007/s11517-007-0298-3

19. Telecommunications Bureau of the Ministry of Internal Affairs and Communications (1990) Radio Wave Protection Guidelines. Telecommunication Technology Council Report: Consultation No. 38th Radio Wave Use and Protection Guidelines for the Human Body (Japanese). https://www.tele.soumu.go.jp/resource/j/material/dwn/guide38.pdf

20. Lilly, M.D. and Leonard, S. (2015) Pathophysiology of Heart Disease: An Introduction to Cardiovascular Medicine. 6th Edition, Lippincott Williams \& Wilkins, Philadelphia.

21. Sahani, A.K., Shah, M.I., Joseph, J. and Sivaprakasam, M. (2016) Carotid and Jugular Classification in ARTSENS. IEEE Journal of Biomedical and Health Informatics, 20, 440-449. https://doi.org/10.1109/JBHI.2015.2403283 